\title{
NUMERICAL MODELS OF MAGNETIC DISK ACCRETION IN STAR FORMING REGIONS
}

\author{
MICHAEL L. NORMAN AND JAMES M. STONE \\ Department of Astronomy, University of Illinois \\ and National Center for Supercomputing Applications \\ Urbana, Illinois 61801
}

\begin{abstract}
We report on preliminary numerical MHD simulations of magnetic disk accretion surrounding a compact protostar in which the disk and the hydromagnetic wind interact self-consistently. We show that an outflow is indeed produced, but that its properties are sensitive to the assumed initial poloidal magnetic field structure. In particular, we reconcile the apparently disparate models of Pudritz and Norman $(1983,1986)$ and Uchida and Shibata (1985) concerning the mass ejection mechanism.
\end{abstract}

\section{Introduction}

It is now well established observationally that the late stages of star formation are accompanied by energetic outflows over a wide range of protostellar luminosities, and consequently, masses (Lada 1985). The most dramatic examples of these outflows are molecular bipolar outflows and optical jets. A key observation that rules out the possibility that the molecular outflows are radiatively driven, especially in low mass systems, is that their momentum exceeds the momentum of the protostellar radiation field by two to three orders of magnitude (Lada 1985). The possibility that the (observed) ionized protostellar wind drives the molecular outflow can also be ruled on energetic and momentum grounds. The current view is that there is a considerable (unobserved) neutral wind produced either by the protostar or its surrounding accretion disk which sweeps up ambient molecular material into a thin shell located around a wind-filled bipolar cavity.

The most promising mechanism for driving the neutral wind is hydromagnetic mass ejection from the protostellar accretion disk. The detection of molecular tori orbiting the embedded protostars lends credence to this picture. Pudritz and Norman $(1983,1986)$ have constructed an elaborate model for magnetic star formation which attempts to link magnetic breaking and wind ejection in the accretion disk to the bolometric luminosity and mass accretion rate of the central protostar. At the heart of their model is the centrifugally-driven hydromagnetic wind mechanism for jet formation in active galactic nuclei studied by Blandford and Payne (1982; hereafter BP). BP showed for the case of a thin, Keplerian accretion disk, outflow is possible if the poloidal component of the magnetic field makes an angle of less than $60^{\circ}$ with the disk surface.

On the other hand, Uchida and Shibata (1985; hereafter US) have performed numerical simulations of magnetic disk accretion, and find mass ejection even though the magnetic field is initially perpendicular to the disk surface. They explain the outflow is being driven by the poleward relaxation of the highly twisted magnetic field just above the disk surface. Their argument, in essence, is that the process of winding up their initially poloidal magnetic field produces a large toroidal component just above the disk surface which has a magnetic pressure gradient sufficient to cause mass ejection. 
We have undertaken to reconcile these apparently disparate points of view through timedependent numerical MHD simulations which closely match those of US. We find that these points of view can indeed be reconciled, and that both processes occur. We find that mass ejection as described by US occurs in an early transient phase, thereafter a centrifugallydriven outflow ensues along those field lines which satisfy the geometrical constraint of BP. Our study emphasizes the importance of modeling the long-term evolution of the magnetic field in both the disk and wind regions, which is an exceedingly challenging problem numerically.

\section{Simulations}

We numerically solve the time-dependent equations of ideal MHD (i.e., assuming flux freezing) in spherical polar coordinates assuming axisymmetry for a thin, Keplerian accretion disk initially threaded by a uniform axial magnetic field. Despite the reduction to two spatial coordinates, all three components of the velocity and magnetic fields are retained as the poloidal and toroidal components of $\mathrm{V}$ and $\mathrm{B}$ couple strongly. The problem we consider is identical to that studied by US (however they used cylindrical coordinates), which is characterized by four dimensionless parameters: $\zeta=v_{\phi} t_{0} / r_{0}=1 ; \eta=v_{s}^{2} / \gamma v_{\phi}{ }^{2}=$ $2.9 \times 10^{-3} ; \xi=\mathrm{v}_{\mathrm{K}}{ }^{2} / \mathrm{v}_{\phi}{ }^{2}=1.6 ; \delta=\mathrm{v}_{A}{ }^{2} / \mathrm{v}_{\phi}{ }^{2}=4.7 \times 10^{-3}$. Here all variables subscripted with "o" are measured at the reference radius, which is taken to be the outer radius of the computation, and $\gamma, v_{\phi}, v_{s}, v_{K}$, and $v_{A}$ are, respectively, the adiabatic constant $=5 / 3$, the sound speed, the disk rotation speed, the Keplerian rotation speed, and the Alfven speed, all evaluated at the reference radius. The accretion disk is embedded in a hot, spherically symmetric corona which is in hydrostatic equilibrium with respect to a gravitational point source at $r=0$. The computational domain comprises $0.1 \leq r / r_{0} \leq 1$, and $0 \leq \theta \leq \pi / 2$, which is spanned by 40 logarithmically ratioed radial zones (ratio=1.04) and 40 uniform angular zones.

The simulations were performed using the ZEUS-2D code developed by the authors, which incorporates the hydrodynamic algorithms described in Norman and Winkler (1986) as well as the CT method of Evans and Hawley (1988) for magnetic field evolution. ZEUS2D is a time-explicit, Eulerian ideal MHD code which has been tested against a battery of problems including the 1-D magnetic Riemann problem of Brio and Wu (1988), a 1-D Weber-Davis (1967) wind solution, and the 2-D solar transient solution of Low (1984).

\section{Results}

Figure 1 shows density distribution and the poloidal magnetic field lines for the parameter set given above at dynamical time $t / t_{0}=1$. The bulge in the density isocontours in the inner regions of the disk indicate a region of mass ejection. A typical velocity in this feature is 2 in dimensionless units, which exceeds the local escape velocity of $3^{1 / 2}$. The figure shows that the poloidal magnetic field lines make a significant angle with respect to the rotation axis even though they are initially parallel. This is because the disk rotation is chosen to be initially sub-Keplerian ( $\xi=1.6)$ following US; as disk material subsequently settles into lower orbits, it drags the magnetic field in with it producing the observed distortion.

Figure 1 is in good qualitative agreement with the results of US (cf. Fig 3, t=1.9). However we disagree with their interpretation of the mass ejection mechanism, which they ascribe to the transient relaxation of toroidal magnetic pressure as summarized above. Rather, we believe that we are seeing a quasi-steady centrifugally-driven wind of the sort 


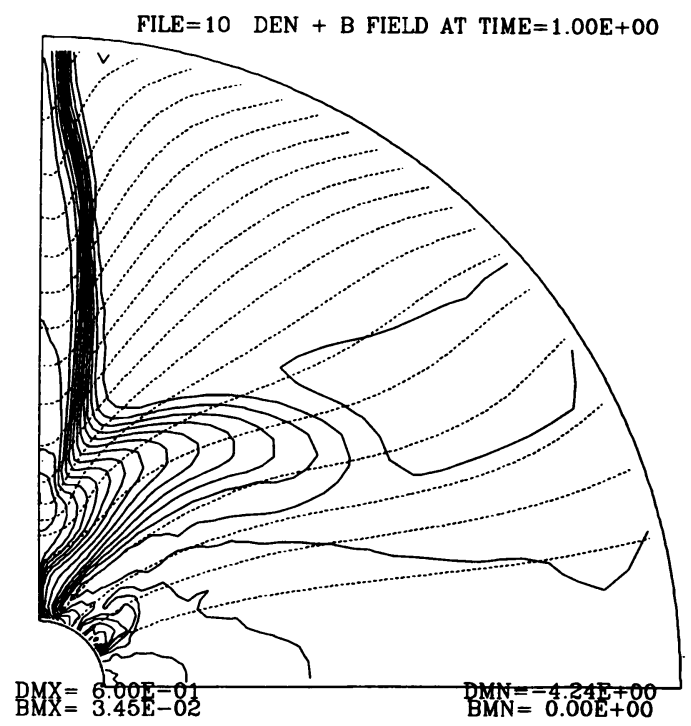

Fig. 1 Density isocontours (solid) and poloidal magnetic field lines (dotted) showing hydromagnetic outflow from an initially sub-Keplerian accretion disk at $t / t_{0}=1$ (disk edge has rotated through one radian). Axis is horizontal and equator is vertical.

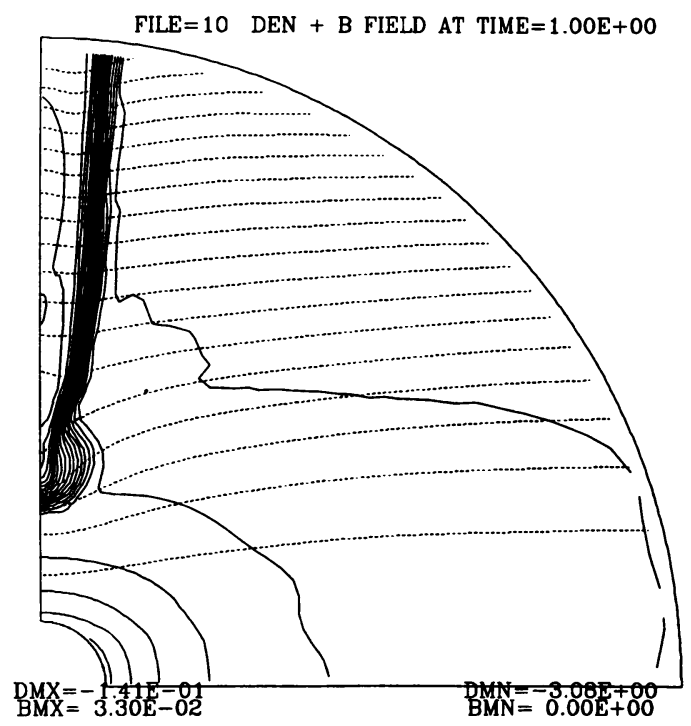

Fig. 2 Same display as in Fig. 1 showing lack of hydromagnetic outflow from an initially Keplerian accretion disk at $t / t_{0}=1$. Note the considerable difference in magnetic field geometry with respect to Fig. 1, whose importance is explained in the text. 
analyzed by BP. To test this conjecture, we repeated the calculation with a Keplerian disk in centrifugal balance and all other parameters held fixed. The results of this calculation are shown in Fig. 2. As can be clearly seen, the plume of ejecta is absent. Note that nowhere are the magnetic field lines inclined less than $60^{\circ}$ to the disk normal as required for centrifugally-driven outflow.

\section{Implications}

We have shown that the properties, and indeed, the existence of the hydromagnetic outflow is sensitive to the magnetic field orientation where it emerges from the disk surface. In particular, outflow is found to occur on those field lines which roughly satisfy the geometrical constraint $\left[\tan ^{-1}\left(\mathrm{~B}_{\mathrm{Z}} / \mathrm{B}_{\mathrm{r}}\right)<60^{\circ}\right]$ derived by $\mathrm{BP}$. The late time outflow produced in the simulations of US can be understood as a consequence of their assumed sub-Keplerian initial conditions for the disk, which produces favorably oriented magnetic field lines as the disk adjusts to centrifugal equilibrium.

It is hard to imagine how such a dynamic readjustment could arise during the quasi-static contraction of the molecular cloud via ambipolar diffusion as is currently envisioned (Mouschovias 1987). However, as shown by Tomisaka et al. (1988), when the mass-toflux ratio exceeds a critical value, equilibrium is no longer possible and gravitational collapse ensues. It is clearly important to follow this collapse to ascertain which magnetic geometries are produced in the resulting molecular disk. We are planning simulations to address this dynamic phase, which are entirely feasible with our current numerical code. The calculation is simplified since one may ignore ambipolar diffusion effects as the collapse timescale is much shorter than the diffusion timescale. After centrifugal equilibrium is established, however, ambipolar diffusion is again important as its timescale is now shorter than the accretion timescale. Simulating this phase will be extremely challenging computationally, as implicit techniques will be required. At some point, the protostar will begin to influence the ionization and temperature structure of the inner portions of the accretion disk, requiring the inclusion of radiative transfer. Prospects for incorporating this physics into ZEUS-2D is discussed by Stone et al. (1989).

\section{Acknowledgements}

The calculations presented here were performed on the CRAY X/MP system at the National Center for Supercomputing Applications, University of Illinois, Urbana-Champaign.

\section{References}

Brio, M. and Wu, C. 1988, J. Comp. Phys. 75, 400.

Blandford, R. and Payne, D. 1982, M.N.R.A.S., 199, 883.

Evans, C. and Hawley, J. 1988, Astrophys. J., 332, 659.

Lada, C.J. 1985, Ann. Rev. Astron. Astrophys., 23, 267.

Low, B.C. 1984, Astrophys. J., 281, 392.

Mouschovias, T. 1987, in Galactic and Extragalactic Star Formation, ed. R. Pudritz, (Kluwer Dordrecht), 400.

Norman, M.L. and Winkler, K.-H.A. 1986, in Astrophysical Radiation Hydrodynamics, eds. K.-H. Winkler \& M.L. Norman, (Reidel: Dordrecht),187.

Pudritz, R. and Norman, C. 1983, Astrophys. J., 274, 677. 1986, Astrophys. J., 301, 571.

Stone, J., Norman, M. and Mihalas, D. 1989, in Accretion Disks and Magnetic Fields in Astrophysics, ed. G. Belvedere, (Kluwer: Dordrecht), 207.

Tomisaka, K., Ikeuchi, S. and Nakamura, T. 1988, Astrophys. J., 335, 239.

Uchida, Y. and Shibata, K. 1985, Publ. Astron. Soc. Japan, 37, 515. 
PUDRITZ: Your simulations are very beautiful! After the transient phase ends, one should pick up the presence of the steady centrifugally driven wind phase. The Uchida and Shibata work started to show this. Have you continued your calculations long enough to pick up this phase?

M.L. NORMAN: We believe we are seeing the beginning of this phase near the end of the simulation shown. Numerical difficulties prevented carrying the calculation to greater dynamical times. We will be working to overcome these difficulties in the coming months, with the interest of seeing whether a steady-state flow is achieved.

KUNDT: Through what distances do you expect your jets to remain focussed? (My reservations are expressed, e.g., in Blome and Kundt (1988, Astrophys. Space Sci. 148, 343).

M.L. NORMAN: In all steady hydromagnetic wind solutions studied thus far by Sakurai, a high degree of collimation is insured for sufficiently large distances above the disk since they carry a net current and toroidal field which produce an inward $\nabla \times B$ force. Shocks and nonaxisymmetric instabilities may destroy jets before they become well-collimated. Future simulations will address these questions.

PERATT: In the laboratory, magnetized hollow jets or beams often suffer filamentation and beam breakup instabilities as they propagate away from the source. Do your simulations show this phenomena, or is a threedimensional approach required?

M.L. NORMAN: Two-dimensional simulations cannot study such azimuthal instabilities. We are currently developing a three-dimensional MHD code for this purpose.

CAMENZIND: Plasma in accretion disks has a finite conductivity. To what extent does the distribution of magnetic fields depend on a finite magnetic diffusivity and on the numerical effects?

M.L. NORMAN: We will be incorporating a phenomenological resistivity in the disk to address this question.

KOUPELIS: (a) Is there a special reason for using the form of the energy equation that describes only the evolution of the internal energy instead of using the full energy equation which includes the magnetic terms?

(b) I realize that the new code will be able to satisfy the $\vec{\nabla} \cdot \vec{B}=0$ equation. Could you tell us how was this problem resolved?

M.L. NORMAN: (a) Total energy formulations produce inaccurate estimates of the internal energy in regions where it is a small fraction of the total energy, such as in the low $\beta\left(\equiv \mathrm{P} /\left(\mathrm{B}^{2} / 8 \pi\right)\right)$ magnetized wind zone. In ideal flows, this is normally not a problem, however when including radiation, accurate temperatures are needed to evaluate the source function, opacities, etc.

(b) We use the constrained transport method of Evans and Hawley (1988, 
Astrophys. J. 322, 659), which insures $\nabla . B=0$ to roundoff while allowing one to evolve the magnetic field components directly. We find this method to be more accurate than ones based on the poloidal flux function, since only first differences are required to evaluate the current density.

PUDRITZ: The criticism of Shu (1985, IAU Symp. No. 115) and Shu et al. (1987, Ann. Rev. Astron. Astrophys.) of the model by Pudritz (1985, Astrophys. J. 293, 216) and Pudritz and Norman (1986, Astrophys. J. 301, $571)$ is incorrect. In Pudritz (1985) the scenario was developed for very massive star formation ( $M_{c o r e}=10^{2} M_{\odot}, L_{I R}=10^{5} L_{\odot}, R_{d}=10^{17} \mathrm{~cm}$ ) as for a $30 \mathrm{M}_{\odot}$ star. Recently Bally et al. have found such massive objects. The criticism of Shu, to which you refer, was that enormous disks would be needed to account for low mass star formation. This point is incorrect. I have scaled my calculations to regions of low mass star formation such as L155, and find that all known attributes of low mass star formation (bipolar flow energy, $M_{d i s k}<1 M_{\odot}$, wind momentum, $L_{*}$, etc.) are neatly reproduced (see Pudritz, 1988, in Galactic and Extragalactic Star Formation, eds. R.E. Pudritz and M. Fich, Kluwer, Dordrecht). The point is that strong magnetic fields efficiently convert gravitational binding energy of accretion disks into ordered mechanical energy in bipolar flows.

MOUSCHOVIAS: I strongly disagree that Pudritz and Norman (1986) showed that their calculation on mass ejection from a disk surrounding a central star can explain the observations of bipolar outflows. That calculation effectively treated the magnetic field lines as rigid wires anchored in the disk and opening up away from the axis of symmetry (which is also the axis of rotation) in the surrounding medium. Matter, in this configuration, slides like beads along wires under the action of the centrifugal force. So, the faster the disk (and, therefore, the wire) rotates and the larger the angle of the wire with respect to the axis of symmetry, the faster the bead will be ejected. However, this model violates Newton's 3rd law, because it ignores the force that a bead exerts on the wire (i.e. the ejected mass will modify the field lines). In other words, the model must include Faraday's law, $\partial \vec{B} / \partial t=\nabla_{x}(\vec{U} \times \vec{B})$, and determine the evolution of the magnetic field itself simultaneously with the evolution of the velocity field. 